\title{
Produção de açafrão em função de acessos e do peso de rizomas-semente
}

BERNI, R.F."*; CHAVES, F.C.M.1; PINHEIRO, J.B.2; VAZ, A.P.A. ${ }^{3}$

'Embrapa Amazônia Ocidental, Km 29 - AM 010, CP 319, 69.011-970, Manaus - AM. rodrigo.berni@cpaa. embrapa.br; '2Escola Superior de Agricultura Luiz de Queiróz (ESALQ), Avenida Pádua Dias, 11 - 13418-900, Piracicaba/SP; ' ${ }^{E}$ mbrapa Transferência de Tecnologia, Escritório de Negócios de Campinas Avenida Dr. André Tosello, 209, Cidade Universitária, CP: 6062,13083-970, Campinas, SP.

RESUMO: O açafrão-da-terra (Curcuma longa L.) é originário do sudeste asiático e subcontinente indiano. É uma herbácea de caule subterrâneo, alaranjado, com vários rizomas secundários aproveitados na indústria alimentícia e farmacêutica devido às características de cor, sabor, odor, produção de óleos essenciais, e corantes. Na escolha do melhor material propagativo deve-se levar em consideração o material genético, o peso, tamanho, idade, capacidade de reserva acumulada, sanidade, dentre outros fatores. O objetivo neste experimento foi avaliar a influencia de diferentes acessos e pesos de rizomas-sementes na produção de açafrão. Empregou-se o delineamento em blocos casualizados em parcelas subdivididas com quatro repetições, tendo como tratamento principal os acessos (C-06, C-36 e C-38) e como tratamento secundário as classes de rizomas-sementes (peso): pequeno, médio e grande, $\pm 5, \pm 10$ e $\pm 15 \mathrm{~g} /$ rizoma, respectivamente. Após a colheita, os rizomas foram distribuídos sobre tela suspensa para secagem à sombra com ventilação natural, por 20 dias. Posteriormente, para avaliar a produção, os rizomas foram classificados em 4 classes: A (> 15g ), B $( \pm 10 \mathrm{~g}), \mathrm{C}( \pm 5$ g) e $D(<5 g)$. A interação entre os acessos e o tamanho do rizoma-semente foi significativa para todas as variáveis, com exceção da Classe D. Com o uso de rizoma-semente grande os acessos C-38 e o C-06 apresentaram maior produtividade total, 0,834 e 0,812kg/planta, respectivamente. O descarte gerado foi menor no acesso C-38 do que no C-06, representando 7,8 e $12,8 \%$ da produção total, respectivamente. O uso de rizomas-semente maiores aumentou significativamente a produção total. No acesso C-06 a produção passou de 0,481 para 0,812 $\mathrm{kg} /$ planta, ou seja, um aumento de $70 \%$. O ganho relativo na produção de rizoma (kg/planta) no acesso C-06 para o plantio de rizomas com $\pm 15 \mathrm{~g}$, foi de $28 \%$.

Palavras-chave: Curcuma longa L., propagação vegetativa, classificação de rizomas, Amazônia

ABSTRACT: Influence of the weight of seed-rhizomes in the production of turmeric. Turmeric (Curcuma longa L) originated in Southeast Asia and the Indian subcontinent. It is an herbaceous plant with underground, orange stem with several secondary rhizomes used in the food and pharmaceutical industries, because of its characteristics of color, taste, smell, production of essential oils and dyes. In cultivation, the best choice of propagation material must take account the genetic material, weight, age, accumulated reserve capacity, sanity, among other factors. In this study, seed-rhizomes of three weight categories - small, medium and large $-, \pm 05, \pm 10$ and $\pm 15 \mathrm{~g} /$ rhizome, respectively, of three genetic materials - C-06, C-36 and C-38 - from the Germplasm Bank of the ESALQ / USP were cultured from December 2009 to August 2010, at a spacing of $0.5 \mathrm{mx} 0.2 \mathrm{~m}$. After harvest, they were distributed on canvas suspended for drying in the shade and natural ventilation for 20 days. Later, to evaluate the production, they were classified into four categories: A (>15g), B $( \pm 10 \mathrm{~g}), \mathrm{C}( \pm 5 \mathrm{~g})$ and D $(<5 \mathrm{~g})$ .The interaction between accessions and size of seed-rhizomes was significant for all variables, except for category D. With the use of large seed-rhizomes, C-38 and C-06 had a higher total yield, with 0.834 and $0.812 \mathrm{~kg} / \mathrm{plant}$, respectively. The use of large seed-rhizomes increased significantly the total production. In C-06, the production increased from 0.481 to $0.812 \mathrm{~kg} / \mathrm{plant}$, i.e. an increase of $70 \%$. Also in C-06, the relative gain in the production of rhizome ( $\mathrm{kg} / \mathrm{plant}$ ) for the planting of seed-rhizomes with $\pm 15 \mathrm{~g}$ was $28 \%$.

Key words: Curcuma longa L., vegetative propagation, rhizome classification, Amazon 


\section{INTRODUÇÃO}

O açafrão-da-terra ou cúrcuma (Curcuma longa L.), da família Zingiberaceae, tem origem no sudeste da Ásia e subcontinente indiano. Planta herbácea, $120-150 \mathrm{~cm}$ de altura; rizomas tuberculosos, o central alongado e os laterais arrendondados, duros, de cor variável interiormente, predominando a laranjada; folhas, grandes (pecíolo tão comprido como o limbo), oblongo-lanceoladas, reunidas na base, oblíquo-nervadas, aromáticas quando contusas; flores amareladas, longopedunculadas, dispostas em espigas compridas e com brácteas côncavas verde-pálidas, as superiores também com uma mancha rósea; fruto capsula bivalve, com três lóculos. Os rizomas encerram amido, um óleo essencial (cineol, felandreno), duas matérias tintoriais, sendo uma parda, geralmente desprezada e outra amarela e resinosa, que é a curcumina, muito empregada na tinturaria e na alimentação humana (Correa,1926). É cultivado atualmente na Ásia sub-oriental, Índia, Jamaica, Peru e Haiti. No Brasil, a espécie foi introduzida pelos bandeirantes para marcar trilhas das minerações. Devido às suas características de cor, sabor e odor, é considerada uma preciosa especiaria, por compor famosos temperos, entre eles o "curry". Tem-se também a produção de óleos essenciais e corantes, muito aproveitados na indústria alimentícia e farmacêutica (Govindarajan, 1980; Goto, 1993). Diversas propriedades medicinais são atribuídas, tais como: antiinflamatório, antibacteriana, antiimuno-deficiência viral humana, efeitos antioxidantes e atividade nematicida (Araújo \& Leon, 2001).

Embora a maior região produtora esteja localizada no Centro-Oeste do país, onde segundo Milhomem e Teixeira (1999) é de grande importância socioeconômica para a agricultura familiar, é na região Norte que o uso do açafrão é mais difundido, sendo utilizado para dar cor amarelada ao arroz e farinha de mandioca. Cecílio Filho et al.(2000) reforçam a importância da cúrcuma como opção aos produtores e do segmento agroindustrial, mas ressaltam que para aumentar a adoção da matéria-prima, temse a necessidade de pesquisas de pós-colheita, armazenamento, processamento e uso. Milhomem e Teixeira (1999) destacam que embora a cultura tenha o potencial para produzir 50 ton/ha de rizoma verde, na região de Mara Rosa (GO) a média é de 16,5 ton/ha. As estatísticas mais recentes sobre a cultura do açafrão constam no Censo Agropecuário de 1995 (IBGE, 2013), onde a produção total brasileira foi de 4.486 toneladas e os cinco municípios com maior contribuição foram: Mara Rosa/GO (23,8\%), Ibirité/MG (15,9\%), Suzano/ SP $(10,7 \%)$, Mogi das Cruzes/SP (8,54\%) e Santo
Antônio do Paraíso/PR (2,9\%).

Informações que auxiliem na identificação de diferenças de respostas entre germoplasmas são interessantes, pois foi relatado que a divergência genética nas coleções de cúrcuma existentes no Brasil é muito baixa e foram identificados, por descritores morfo-agronômicos, somente 4 grupos distintos (Sigrist et al., 2011), o que auxilia nas estratégias para diminuir a proximidade genética das coleções.

No cultivo, a escolha do melhor material propagativo, quer seja semente ou estruturas vegetativas deve levar em consideração o material genético, o peso, tamanho, idade, capacidade de reserva acumulada, sanidade, dentre outros fatores. Menezes et al. (2005) verificaram que o tipo de rizoma semente de açafrão influencia no rendimento do cultivo, ocorrendo um acréscimo de $30 \%$ na biomassa com o uso do rizoma do tipo "cabeça" (rizoma central, principal) quando comparado ao uso dos rizomas semente do tipo "dedo"(rizoma secundário). Chaves et al. (2000) verificaram que os melhores rizomas-sementes foram aqueles com peso médio superior a 7,0 g e Maia et al. (1995) relataram que o potencial de produção das plantas originadas de rizomas primários (massa média de $19 \mathrm{~g}$ ) chegam a superar em $50 \%$ ao das plantas originadas de rizomas secundários (massa média de 9g).

Em função do exposto, o objetivo deste trabalho foi avaliar o efeito sobre a produção, do uso de rizomas-semente, classificados pelo peso, de três acessos de açafrão, cultivados nas condições de Manaus, AM.

\section{MATERIAL E MÉTODO}

O experimento foi realizado no Setor de Plantas Medicinais e Hortaliças da Embrapa Amazônia Ocidental, Manaus, AM, no período de dezembro de 2009 a agosto de 2010, em área localizada aos $02^{\circ} 53^{\prime} 24^{\prime \prime} S$ e $59^{\circ} 58^{\prime} 00^{\prime \prime} \mathrm{W}$ e $108 \mathrm{~m}$ de altitude, em Latossolo Amarelo Argiloso, com os seguintes resultados obtidos da análise química: $\mathrm{pH}\left(\mathrm{H}_{2} \mathrm{O}\right)=5,41 ; \mathrm{P}_{\text {menhich }}=3 \mathrm{mg} \mathrm{dm}^{-3}$; matéria orgânica $=37 \mathrm{~g} \mathrm{~kg}^{-3} ; \mathrm{V} \%=21 ; \mathrm{Al}=0,36 \mathrm{cmolc} \mathrm{dm}^{-3}$; $\mathrm{H}+\mathrm{Al}=4,32 \mathrm{cmolc} \mathrm{dm}^{-3} ; \mathrm{K}=18 \mathrm{mg} \mathrm{dm}^{-3} ; \mathrm{Ca}=0,82$ $\mathrm{cmol}_{\mathrm{c}} \mathrm{dm}^{-3} ; \mathrm{Mg}=0,28 \mathrm{cmol}_{\mathrm{c}} \mathrm{dm}^{-3} ; \mathrm{SB}=1,15 \mathrm{cmol}_{\mathrm{c}}$ $\mathrm{dm}^{-3} ; \mathrm{CTC}=5,48 \mathrm{cmol}_{\mathrm{c}} \mathrm{dm}^{-3}, \mathrm{Fe}=130 \mathrm{mg} \mathrm{dm}^{-3}$; $\mathrm{Zn}=0,75 \mathrm{mg} \mathrm{dm}^{-3} ; \mathrm{Mn}=3,08 \mathrm{mg} \mathrm{dm}^{-3}$ e $\mathrm{Cu}=0,23 \mathrm{mg}$ $\mathrm{dm}^{-3}$. Durante o ensaio a temperatura média foi de $26,9^{\circ} \mathrm{C}$ e a precipitação acumulada foi de $2.095,3$ $\mathrm{mm}$, segundo os dados da Estação Meteorológica da Embrapa Amazônia Ocidental, localizada nas coordenadas $02^{\circ} 53^{\prime} 25^{\prime \prime}$ S e 59 $58^{\prime} 06^{\prime \prime} \mathrm{W}$. Anteriormente à instalação desse ensaio, os

Rev. Bras. PI. Med., Campinas, v.16, n.3, supl. I, p.765-770, 2014. 
rizomas-sementes dos acessos C-06, C-36 e C-38, do Banco Ativo de Germoplasma-BAG da Escola Superior de Agricultura Luiz de Queiroz (ESALQ/ USP) foram multiplicados em área no Campo Experimental da Embrapa Amazônia Ocidental, no período de dezembro de 2008 a agosto de 2009 para o aumento do material propagativo. Após a colheita e beneficiamento, os rizomas foram secos à sombra, com ventilação natural e temperatura ambiente, por 20 dias. A temperatura média durante o período foi de $34,5^{\circ} \mathrm{C}$ e a umidade relativa média do ar de $77,6 \%$. Posteriormente os rizomas foram separados em função do peso médio ( $\mathrm{g} / \mathrm{rizoma}$ ), ficando assim distribuídos: rizomas-sementes de três categorias de peso: pequeno $( \pm 05 \mathrm{~g})$, médio $( \pm 10 \mathrm{~g})$ e grande $( \pm 15 \mathrm{~g} /$ rizoma), tendo um controle por escala comparativa e verificação com aceite de rizomas com no máximo $10 \%$ de variação em cada categoria. Sessenta dias anteriormente ao plantio foram aplicados $1.050 \mathrm{~g}$ de calcário por canteiro $(15 \mathrm{~m} \times 1,0 \mathrm{~m})$. Os rizomas-sementes, por categoria, em número de 10 por parcela, foram plantados a uma profundidade de $5,0 \mathrm{~cm}$, em canteiros com altura de $30,0 \mathrm{~cm}$, adubados com esterco bovino $\left(3,0 \mathrm{~kg} / \mathrm{m}^{2}\right)$ quando do preparo dos mesmos e aos 5 meses após o plantio. Os canteiros tinham $15 \mathrm{~m}$ de comprimento e $1,0 \mathrm{~m}$ de largura, distantes $0,5 \mathrm{~m}$ entre os canteiros.

O delineamento foi em blocos casualizados, em um esquema de parcelas subdivididas, sendo o fator principal os acessos e o tratamento secundário as classes de rizomas-sementes (peso) e quatro repetições. O espaçamento foi de $0,5 \mathrm{~m} \times 0,2 \mathrm{~m}$, tendo duas linhas e 10 plantas na área útil na subparcela. Devido ao regime pluviométrico satisfatório durante o ciclo da cultura, não foi necessário a suplementação de água por irrigação. Foi realizado sempre que necessário a capina manual dos canteiros para redução da competição com as plantas daninhas. Em agosto de 2010, quando a parte aérea estava com folhas acamadas e amarelas, foi feita a colheita aos 240 dias após o plantio. Os rizomas foram distribuídos sobre tela suspensa para secagem à sombra e com ventilação natural, por 20 dias, com um teor de umidade médio de $78 \%$. Posteriormente, para avaliar a produção, os rizomas foram classificados em quatro classes: A (>15g ), B $( \pm 10 \mathrm{~g}), \mathrm{C}( \pm 5 \mathrm{~g})$ e D $(<5 \mathrm{~g})$ (sem valor para mesa - apenas de uso na indústria). Foi também separado o descarte (restos de cultura: raiz, folhas secas, caule). A produção total de rizoma/ planta para uso comercial e beneficiamento, ou seja, sem a separação em classes, foi obtida pela soma de todas as classes, descontado o descarte. A análise de variância dos dados foi realizada no programa IRRISTAT 5.0 e o teste de comparação de médias utilizado foi o de Tukey ao nível de $5 \%$ de probabilidade.

\section{RESULTADO E DISCUSSÃO}

Houve interação significativa entre os acessos e o tamanho do rizoma-semente para todas as variáveis avaliadas, à exceção da produção na classificação D (Tabela 1). Para a variável com interação não significativa, a produção de rizomas na classe $\mathrm{D}$, foi observado que os materiais C-38, C-06 e o C36 produziram 0,198, 0,186 e 0,091 kg/ planta, respectivamente. Ademais o uso de rizomassemente, pequeno, médio e grande, produziram respectivamente 0,$142 ; 0,138$ e $0,196 \mathrm{~kg} /$ planta, sendo significativamente menor a produção do C-06 e maior a produção com o uso de rizomas-semente da categoria grande, resultado similar aos de Maia et al. (1995), que relataram que rizomas com aproximadamente o dobro de massa superaram em $50 \%$ a produção dos demais com metade da massa.

$\mathrm{Na}$ interação envolvendo acessos e cada categoria de rizomas-semente, observa-se que com o uso de rizoma-semente grande $( \pm 15 \mathrm{~g} /$ rizoma), os acessos C-38 e o C-06 apresentaram maior produtividade total, 0,834 e $0,812 \mathrm{~kg} /$ planta, respectivamente (Tabela 2). Em um ensaio anterior (Chaves et al., 2010), a menor produção foi encontrada no acesso C-38, resposta diferente dessa avaliação, constatando que as respostas são diferenciadas na interação ambiente x germoplasma, de um lado uma cultura de ciclo que abrange mais de $65 \%$ do período de um ano, do outro as condições de trópico úmido, que tem períodos distintos de regime hídrico (Af na classificação de Köppen-Geiger).

A produção do C-38, depois de classificada se concentrou, aproximadamente $45 \%(0,376$ de $0,834 \mathrm{~kg} /$ planta), na classificação $C$, enquanto o C-06 teve $52 \%$ da sua produção na classificação $A$ e $B$, que são rizomas maiores. Embora na avaliação de descarte gerado, que reflete no custo do beneficiamento da produção, tenha sido menor no acesso C-38 do que no C-06, representando 7,8 e $12,8 \%$ da produção total, respectivamente.

Em geral observa-se que em todos os acessos, o uso de rizomas-semente maiores (categoria grande) aumentou significativamente a produção total, o que correspondeu ao encontrado por Cecílio Filho \& Souza (1994) e Maia et al.(1995), onde o uso de rizomas-semente maiores proporcionou mais vigor às plantas e ganho em produtividade. No acesso C-06 a produção passou de 0,481 para 0,812 kg/planta, ou seja, um aumento de $70 \%$, enquanto nos outros materiais o aumento foi por volta de $40 \%$, indicando que os materiais podem apresentar respostas diferenciadas ao uso da classificação de rizomas para plantio.

Considerando a média das produções totais (Tabela 2), das categorias de rizomas sementes em cada acesso, para referenciar uma produção em que o produtor não diferenciasse as categorias e

Rev. Bras. PI. Med., Campinas, v.16, n.3, supl. I, p.765-770, 2014. 
TABELA 1. Análise de variância do experimento de avaliação da produção de rizomas de acessos de açafrão, a partir de três categorias de rizomas-semente. Manaus, AM, 2014.

\begin{tabular}{|c|c|c|c|c|c|c|c|}
\hline \multirow{3}{*}{ Causa da Variação } & \multirow[t]{3}{*}{$\mathrm{GL}^{1}$} & \multicolumn{6}{|c|}{ Produção de rizomas (kg/planta) } \\
\hline & & \multicolumn{2}{|c|}{$\begin{array}{c}\text { Classificação A } \\
>15,0 \mathrm{~g}\end{array}$} & \multicolumn{2}{|c|}{$\begin{array}{c}\text { Classificação B } \\
\pm 10,0 \mathrm{~g}\end{array}$} & \multicolumn{2}{|c|}{$\begin{array}{c}\text { Classificação C } \\
\pm 5,0 \mathrm{~g}\end{array}$} \\
\hline & & $\mathrm{QM}^{1}$ & $F^{1}$ & QM & $\mathrm{F}$ & QM & $\mathrm{F}$ \\
\hline Blocos & 3 & 0,1041E-03 & $0,44^{\text {ns }}$ & $0,6226 \mathrm{E}-04$ & $0,7 \mathrm{~ns}$ & $0,7504 \mathrm{E}-03$ & $8,1^{*}$ \\
\hline Acessos(Parcela) & 2 & $0,1692 \mathrm{E}-02$ & $7,2^{*}$ & $0,5797 \mathrm{E}-01$ & $639,3^{* *}$ & $0,2818 \mathrm{E}-01$ & $302,7^{\prime \prime}$ \\
\hline Erro a & 6 & $0,2359 \mathrm{E}-03$ & & $0,9068 \mathrm{E}-04$ & & $0,9312 \mathrm{E}-04$ & \\
\hline $\begin{array}{l}\text { Rizomas-sementes(Sub } \\
\text { parcela) }\end{array}$ & 2 & $0,4594 \mathrm{E}-02$ & $42,5^{*}$ & $0,2586 \mathrm{E}-01$ & $136,8^{* \prime}$ & $0,1378 \mathrm{E}-04$ & $59,8^{* *}$ \\
\hline$A \times B$ & 4 & $0,4254 \mathrm{E}-02$ & $39,3^{*}$ & $0,1255 \mathrm{E}-01$ & $66,4^{*}$ & $0,7266 \mathrm{E}-02$ & $31,5^{\prime \prime}$ \\
\hline Erro b & 18 & $0,1082 \mathrm{E}-03$ & & $0,1891 \mathrm{E}-03$ & & $0,2303 \mathrm{E}-03$ & \\
\hline $\mathrm{CV}^{1}(\%)$ Parcela & & 20,3 & & 6,1 & & 3 & \\
\hline \multirow[t]{3}{*}{ CV(\%) Subparcela } & & 13,7 & & 8,9 & & 5 & \\
\hline & & \multicolumn{2}{|c|}{$\begin{array}{c}\text { Classificação D } \\
<5,0 \mathrm{~g}\end{array}$} & \multicolumn{2}{|c|}{ Produção Total } & \multicolumn{2}{|c|}{$\begin{array}{c}\text { Descarte } \\
\text { (Restos culturais) }\end{array}$} \\
\hline & & QM & $\mathrm{F}$ & QM & $\mathrm{F}$ & QM & $\mathrm{F}$ \\
\hline Blocos & 3 & $0,9557 \mathrm{E}-03$ & $1,53^{\text {ns }}$ & $0,2739 \mathrm{E}-02$ & $1,76^{\text {ns }}$ & $0,5544 \mathrm{E}-04$ & $0,44^{\text {ns }}$ \\
\hline Acessos(Parcela) & 2 & $0,4088 \mathrm{E}-01$ & $64,0^{* *}$ & $0,1583 \mathrm{E}-01$ & $10,16^{*}$ & $0,1237 \mathrm{E}-03$ & $0,98^{\text {ns }}$ \\
\hline Erro a & 6 & $0,6387 \mathrm{E}-03$ & & $0,1558 \mathrm{E}-02$ & & $0,1259 \mathrm{E}-03$ & \\
\hline $\begin{array}{l}\text { Rizomas-sementes(Sub } \\
\text { parcela) }\end{array}$ & 2 & $0,1274 \mathrm{E}-01$ & $19,2 *$ & 1,7195E-01 & $103,3^{* *}$ & $0,2629 \mathrm{E}-02$ & 28,76 \\
\hline$A \times B$ & 4 & $0,1678 \mathrm{E}-02$ & $2,5^{\text {ns }}$ & $0,2339 \mathrm{E}-01$ & $14,0^{* \prime}$ & 0,3999E-02 & 43,72 \\
\hline Erro b & 18 & 0,6626E-03 & & $0,1665 \mathrm{E}-02$ & & $0,9145 \mathrm{E}-04$ & \\
\hline CV(\%) Parcela & & 16,0 & & 6,0 & & 21 & \\
\hline CV(\%) Subparcela & & 16,3 & & 6,2 & & 18 & \\
\hline
\end{tabular}

${ }^{1} \mathrm{GL}=$ graus de liberdade, $\mathrm{QM}=$ quadrado médio e $\mathrm{F}=$ Teste de $\mathrm{F}$, onde números seguidos de * e ** são significativos ao nível de 5 e $1 \%$ de probabilidade, respectivamente. $\mathrm{CV}=$ coeficiente de variação

TABELA 2. Produção total de rizomas e em cada classe de peso, a partir de diferentes materiais genéticos de açafrão e de três categorias de rizomas-semente. Manaus, AM, 2014.

\begin{tabular}{|c|c|c|c|}
\hline \multirow{2}{*}{ Acesso } & \multicolumn{3}{|c|}{ Categorias de Rizoma-semente } \\
\hline & Pequeno $\pm 5,0 \mathrm{~g}$ & Médio $\pm 10,0 \mathrm{~g}$ & Grande $\pm 15,0 \mathrm{~g}$ \\
\hline & \multicolumn{3}{|c|}{ Produção de rizomas classificação $\mathbf{A}(>15,0 \mathrm{~g})^{1}$} \\
\hline C-06 & $0,112 \mathrm{Aa}$ & $0,024 \mathrm{Bb}$ & $0,120 \mathrm{Aa}$ \\
\hline $\mathrm{C}-36$ & $0,067 \mathrm{Bb}$ & $0,075 \mathrm{Ab}$ & 0,099 Aa \\
\hline \multirow[t]{2}{*}{ C-38 } & $0,046 \mathrm{Bb}$ & $0,071 \mathrm{Aa}$ & $0,070 \mathrm{Ba}$ \\
\hline & \multicolumn{3}{|c|}{ Produção de rizomas classificação $( \pm 10,0 \mathrm{~g})^{1}$} \\
\hline C-06 & $0,117 \mathrm{Ac}$ & $0,284 \mathrm{Ab}$ & $0,304 \mathrm{Aa}$ \\
\hline C-36 & $0,112 \mathrm{Aa}$ & $0,096 \mathrm{Ba}$ & $0,114 \mathrm{Ca}$ \\
\hline \multirow[t]{2}{*}{ C-38 } & $0,088 \mathrm{Bb}$ & $0,104 \mathrm{Bb}$ & $0,176 \mathrm{Ba}$ \\
\hline & \multicolumn{3}{|c|}{ Produção de rizomas classificação $\mathbf{C}( \pm 5,0 \mathrm{~g})^{1}$} \\
\hline & & $80570^{0}$ & 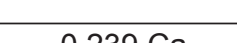 \\
\hline C-06 & $0,192 \mathrm{Cb}$ & $0,227 \mathrm{Ba}$ & 0,239 Ca \\
\hline C-36 & $0,309 \mathrm{Aa}$ & $0,201 \mathrm{Cb}$ & $0,305 \mathrm{Ba}$ \\
\hline C-38 & $0,279 \mathrm{Bb}$ & $0,294 \mathrm{Ab}$ & $0,376 \mathrm{Aa}$ \\
\hline
\end{tabular}

continua...

Rev. Bras. PI. Med., Campinas, v.16, n.3, supl. I, p.765-770, 2014. 
TABELA 2. Produção total de rizomas e em cada classe de peso, a partir de diferentes materiais genéticos de açafrão e de três categorias de rizomas-semente. Manaus, AM, 2014.

continuação...

\begin{tabular}{|c|c|c|c|}
\hline \multicolumn{4}{|c|}{ Produção total de rizomas ${ }^{1}$} \\
\hline C-06 & $0,481 \mathrm{Bc}$ & $0,598 \mathrm{Bb}$ & $0,812 \mathrm{ABa}$ \\
\hline C-36 & $0,666 \mathrm{Ab}$ & $0,528 \mathrm{Cc}$ & $0,742 \mathrm{Ba}$ \\
\hline C-38 & $0,600 \mathrm{Ab}$ & $0,664 \mathrm{Ab}$ & $0,834 \mathrm{Aa}$ \\
\hline \multicolumn{4}{|c|}{$\begin{array}{l}\text { Descarte (restos culturais) }{ }^{1} \\
--\end{array}$} \\
\hline C-06 & $0,016 \mathrm{Cc}$ & $0,037 \mathrm{Ab}$ & $0,104 \mathrm{Aa}$ \\
\hline C-36 & 0,078 Aa & $0,050 \mathrm{Ab}$ & $0,041 \mathrm{Cb}$ \\
\hline C-38 & $0,048 \mathrm{Bab}$ & $0,050 \mathrm{Ab}$ & $0,064 \mathrm{Ba}$ \\
\hline
\end{tabular}

1 Médias seguidas de letras maiúsculas iguais, em cada coluna, e de letras minúsculas iguais, em cada linha, não diferem significativamente entre si pelo teste de Tukey $(p=0,05)$.

TABELA 3. Produção de rizomas e o ganho ou perda de produção de rizomas com o uso de categorias de rizoma-semente, em diferentes materiais genéticos de açafrão, comparativamente à produção média sem o uso de classificação em categorias de rizomas-semente. Manaus, AM, 2014.

\begin{tabular}{|c|c|c|c|}
\hline $\begin{array}{c}\text { Categoria de Rizoma-semente } \\
\text { e Acesso }\end{array}$ & $\begin{array}{c}\text { Produção Média }(A)^{1} \\
\text { (kg/planta) }\end{array}$ & $\begin{array}{c}\text { Produção total por categoria de } \\
\text { rizoma-semente }(\mathrm{B}) \\
(\mathrm{kg} / \text { planta })\end{array}$ & $\begin{array}{c}\text { Ganho(+) ou Perda }(-)^{2} \\
(\%)\end{array}$ \\
\hline \multicolumn{4}{|l|}{ Pequeno $\pm 5,0 \mathrm{~g}$} \\
\hline C-06 & 0,630 & 0,481 & $-23,65$ \\
\hline C-36 & 0,645 & 0,666 & $+3,26$ \\
\hline C-38 & 0,699 & 0,600 & $-14,16$ \\
\hline \multicolumn{4}{|l|}{ Médio $\pm 10,0 \mathrm{~g}$} \\
\hline C-06 & 0,630 & 0,598 & $-5,08$ \\
\hline C-36 & 0,645 & 0,528 & $-18,14$ \\
\hline C-38 & 0,699 & 0,664 & $-5,01$ \\
\hline \multicolumn{4}{|l|}{ Grande $\pm 15,0 \mathrm{~g}$} \\
\hline C-06 & 0,630 & 0.812 & $+28,89$ \\
\hline C-36 & 0,645 & 0,742 & $+15,04$ \\
\hline $\mathrm{C}-38$ & 0,699 & 0,834 & $+19,31$ \\
\hline
\end{tabular}

${ }_{1}$ Produção de rizomas considerando a média das três categorias de rizomas-semente

2 Ganho ou perda percentual da produção $(B)$ com o uso do rizoma-semente classificado em relação à produção média, onde: Ganho ou Perda $=[(A-B) /(A)]^{\star} 100$

plantasse na mesma área uma mistura igualmente distribuída das categorias de rizomas-semente de todos os tamanhos, tem-se em relação à produção média das três categorias um aumento do 15,04; 19,31 e $28,89 \%$ com o uso da categoria grande $(>15 \mathrm{~g})$ dos rizomas-semente para os acessos C-36, C-38 e C-06, respectivamente (Tabela 3). Por outro lado, esse produtor poderia perder até $23,65 \%$ da produção média se usasse a categoria pequeno $( \pm 5,0 \mathrm{~g})$ dos rizomas-semente, no plantio do acesso C-06. Os ganhos obtidos evidenciam a necessidade do produtor realizar a classificação do seu material para o plantio, pois os rizomas-semente maiores apresentam mais reservas para o estabelecimento das plantas, que são mais vigorosas, com maior número de folhas, peso e acúmulo de massa seca (Manhas \& Gill, 2010). Ao considerarmos os custos do uso do rizoma grande tem-se um acréscimo de $300 \%$ em massa de rizoma e provavelmente um maior gasto com o transporte para o plantio da mesma área, mas partindo do exemplo dos resultados de produção do acesso C-38 (Tabela 3), o uso do rizoma pequeno representaria $0,8 \%$ da produção e o uso do rizoma grande representaria

Rev. Bras. PI. Med., Campinas, v.16, n.3, supl. I, p.765-770, 2014. 
$1,8 \%$ da produção de cada planta.

De acordo com os resultados, conclui-se que as combinações que proporcionam as maiores produções são o uso dos acessos C-38 ou C-06, com o plantio de rizomas-semente grandes.

\section{AGRADECIMENTO}

Fonte Financiadora: EMBRAPA e ao convênio FINEP/FAPEAM/FDB No. 01.06.0380.00 - CTIAFAM

\section{REFERÊNCIA}

ARAÚJO, C.A.C.; LEON, L.L. Biological activities of Curcuma longa L. Memórias do Instituto Oswaldo Cruz, Rio de Janeiro, v.96, n.5, p. 723-728, 2001.

CECÍLIO FILHO, A.B.; SOUZA, R.J. de. Influência do espaçamento e peso do rizoma semente na cultura do açafroeiro-da-Índia. Horticultura Brasileira, v.12, p. 76, 1994.

CHAVES, F.C.M.; LAURA, V.A.; QUIJANO, F.G.; CÂMARA, F.L.A. Efeito do peso de rizomas-sementes e de doses de IBA no desenvolvimento de cúrcuma. Horticultura Brasileira, v.18, Suplemento. 2000.

CHAVES, F.C.M.; LIMA, S.C.S.; PINHEIRO, J.B.; FIGUEIRA, G.M.; VAZ, A.P.A. Avaliação agronômica de acessos de açafrão (Curcuma longa) nas condições de Manaus, AM. In: I Congresso Brasileiro de Recursos Genéticos; Workshop em Bioprospecção e Conservação de Plantas Nativas do Semi-Árido; Workshop Internacional sobre Bionergia e MeioAmbiente, 2010, Salvador, BA. Anais.... I Congresso
Brasileiro de Recursos Genéticos; Workshop em Bioprospecção e Conservação de Plantas Nativas do Semi-Árido; Workshop Internacional sobre Bionergia e Meio-Ambiente. 2010. p. 304-304.

CORREA, M.P. Dicionário das plantas úteis do Brasil e das exóticas cultivadas. Rio de Janeiro, Imprensa Nacional, v. 1, p.21-22., 1926.

GOTO, R. Épocas de plantio, adubação fosfatada e unidades térmicas em cultura de açafrão (Curcuma longa L.). 1993. 93f. Tese (Doutorado em Fitotecnia) Universidade Estadual Paulista, Jaboticabal.

GOVINDARAJAN, V.S. Turmeric-chemistry, technology and quality. Critical Reviews in Food Science and Nutrition, v.12, n.3, p.199-301, 1980.

IBGE. Instituto Brasileiro de Geografia e Estatística Sidra. 1995. Censo Agropecuário 1995 - Horticultura. Disponível em: http://www.sidra.ibge.gov.br/bda/tabela/ listabl.asp?z=t\&o=1\&i=P\&e=|\&c=524. Acesso em 25 de fevereiro de 2014.

MAIA, N.B.; BOVI, O.A.; DUARTE, F.R.; SORIA, L.G.; ALMEIDA, J.A.R. de. Influência de tipos de rizomas de multiplicação no crescimento de cúrcuma. Bragantia, 54:33-37, 1995.

MANHAS, S.S.; GILL, B.S. Effect of planting materials, mulch leves and farmayard manure on growth, yield and quality of turmeric (Curcuma longa). Indian Journal of Agricultural Sciences, 80:501-506, 2010.

MENEZES JR., A; BORELLA, J.C.; FRANÇA, S.C.; MASCA, M.G.C.C. Efeitos do tipo de rizoma de multiplicação e da cobertura morta no desenvolvimento e produtividade de cúrcuma (Curcuma longa L.). Revista Brasileira de Plantas Medicinais, 8(1):30-34, 2005.

MILHOMEM, A. de V.; TEIXEIRA, S.M. A cultura do açafrão (Curcuma longa L.) em Goiás - contribuição para a sustentabilidade. Pesquisa Agropecuária Tropical, 29:81-87, 1999. 